\title{
espressoMaths: a drop-by station
}

Karen Henderson

Department of Engineering Design and Mathematics

University of the West of England, Bristol

karen.henderson@uwe.ac.uk

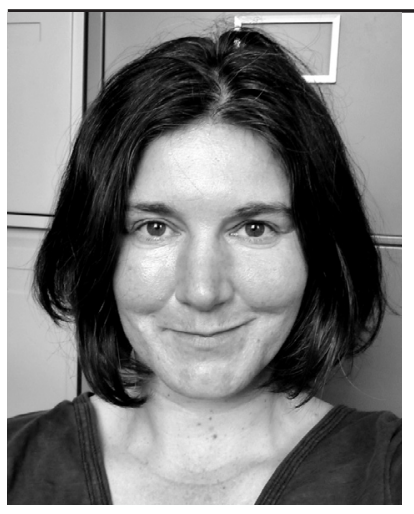

Tim Swift

Department of Engineering Design

and Mathematics

University of the West of England, Bristol tim.swift@uwe.ac.uk

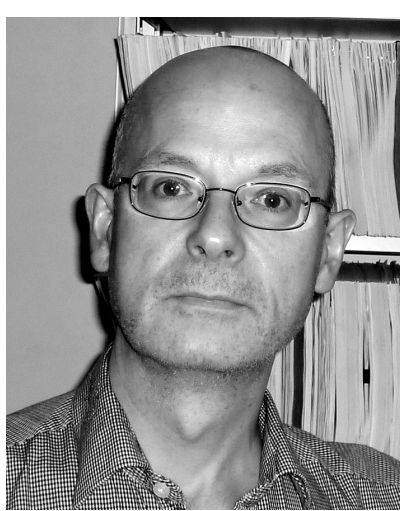

\begin{abstract}
The aim of this short report is to discuss some issues associated with the establishment of, the running of, and the development of a Mathematics and Statistics support drop-by station in a large, distributed campus university environment. The discussion is rooted in our experiences of setting up and of developing espressoMaths at the University of the West of England, Bristol. The main thrust of this contribution is connected with practical rather than with theoretical aspects, although we do touch on some pedagogical issues associated with the interaction between drop-by stations and the dynamics of mainstream teaching and learning.
\end{abstract}

A key intention is to contribute to the ongoing programme of sharing good practice in support structures. An important message is that Mathematics and Statistics support should be liberated from enclosed environments, and it should be open and available to all at the heart of university communities.

\section{Introduction}

Originally inspired by the Maths Café support unit at the University of Portsmouth [1], the drop-by station espressoMaths was developed by a small team of staff in the Department of Mathematics and Statistics at the University of the West of England, Bristol (UWE) over the summer of 2008, and it started operations in September of that year. Since then, espressoMaths has become the primary focus of Mathematics and Statistics support at UWE, a large, distributed campus university. Although it is open to all students and to all university employees, currently the majority of interactions at the drop-by station involve students, rather than staff. Students benefitting from espressoMaths advice come from a wide variety of undergraduate and postgraduate awards in many disciplines.

Although the Department of Mathematics and Statistics at UWE provided Mathematics and Statistics support before 2008, this was within the context of a traditional, more structured, 'behind closed doors' environment. The central idea of the drop-by station philosophy is that support should be available to all members of the university community, whether or not they are attached to modules directly involving Mathematics or Statistics, and that this support should be free, easily accessible, and centrally located in an open space. The emphasis is on 'drop-by', rather than 'drop-in', there are no booked appointments, and there is minimal structure. Interactions should be informal, yet informative.

The main aim of this article is to discuss some issues associated with the establishment of, the running of, and the development of a drop-by station such as espressoMaths. 
We also mention the way in which such a support unit can interact with mainstream teaching and learning.

The themes addressed include the following: good practice regarding the process of setting up and of maintaining a drop-by station; approaches to providing user-friendly advice to the broadest spectrum of users; integrating drop-by stations with other learning resources; marketing, publicity, monitoring and evaluation; dealing with homework, coursework and dissertations; interaction with other university learning spaces and liaison with support services; development and expansion of provision.

\section{Context}

For reference, and in order to establish the context within which espressoMaths operates, the following brief description of UWE may be of interest.

Located in the city of Bristol in south-west England, UWE is a large, post-1992 university with approximately 30,000 students, more than $85 \%$ of whom are from state schools. There are currently around 3,000 academic staff. The main campus is five miles north of Bristol city centre at Frenchay, whilst three other smaller campuses - Glenside, St Matthias and Bower Ashton - are located in other parts of the city.

In addition to the Mathematics and Statistics degree programmes, UWE courses offered within the following disciplines also involve some quantitative elements: Accountancy; Architecture; Business; Computer Science; Construction; Economics; Education; Engineering; Games Technology; Geography; Health Professions; Life Sciences; Marketing; Psychology; Social Science; Surveying. Students from most of these areas have availed themselves of espressoMaths support and advice since the inception of the drop-by station.

\section{Development}

For many years, UWE has had in place various extracurricular means of supporting students in Mathematics and Statistics. In the years immediately preceding the introduction of espressoMaths, this support was based around the Mathematics Resource Centre, a room located within the faculty which is equipped with working space, PCs and a small library of textbooks. This room was used for drop-in sessions, both informal and formal, and also as a space within which students could undertake self-study, either individually or in small groups. The use of this room met some success as a drop-in centre. However, the noncentral location of the Mathematics Resource Centre, and also the fact that entry is via a closed door (requiring the use of a swipe card), meant that many students seeking help with mathematical or statistical problems perceived a barrier - geographical, physical or psychological - between themselves and the source of advice or support. Motivated by a desire to integrate Mathematics and Statistics support into the heart of the university community, and influenced by the success of Maths Café [1], it was decided to introduce a drop-by station at UWE.

The development of espressoMaths involved the following components: choosing a name; choosing a location; interacting with non-academic colleagues such as those in catering, graphic design and student support; designing and disseminating publicity materials; deciding on the frequency and staffing of the drop-by station.

Regarding location, it was decided to use a table in OneZone, the large, busy central refectory on Frenchay campus. This is a central, easily accessible space, which is available to the entire university community. This location proved to be a popular one with Frenchay users of the service, and it is still used today. Regarding frequency, we staff espressoMaths for two hours in the middle of the day, and on every weekday during teaching weeks. The OneZone service is now in its third year of operation. Since September 2010, we have also run a drop-by station on the smaller Glenside campus (situated about two miles from the Frenchay campus), thereby facilitating the availability of support for students and staff in Glensidebased disciplines; these include allied health professions, social sciences, nursing and midwifery. The Glenside service is located at a table in Traders, the bustling refectory on that campus, and, currently, it is staffed on Tuesdays and Thursdays during teaching weeks for two hours in the middle of the day.

Publicity drives have used various media, and they have been targeted at specific events, in specific locations, and at specific classes of students and of staff. Specific events include Induction Week, Freshers' Fair, orientation meetings, and the International Students Welcome, whilst specific places include the Student Village, the Library, and student support areas. Publicity media include the following: posters (e.g., see Fig 1 overleaf); postcards; business cards; pens; monitor screen announcements; student newspaper articles; email shots; internet. Note that internet access is available directly at the espressoMaths homepage [2], and also via the University mySkills page [3]. The original design of the website was put together by a group of Information Systems students as a part of their final year dissertation work.

Module leaders across the University have been encouraged to include reference to espressoMaths in their module handbooks, and in their virtual learning environments. In addition to teaching staff, we have ensured that the following classes of colleagues have been kept informed about espressoMaths activity: programme leaders; student advisers; study skills advisers; library services staff; students' union officers. 


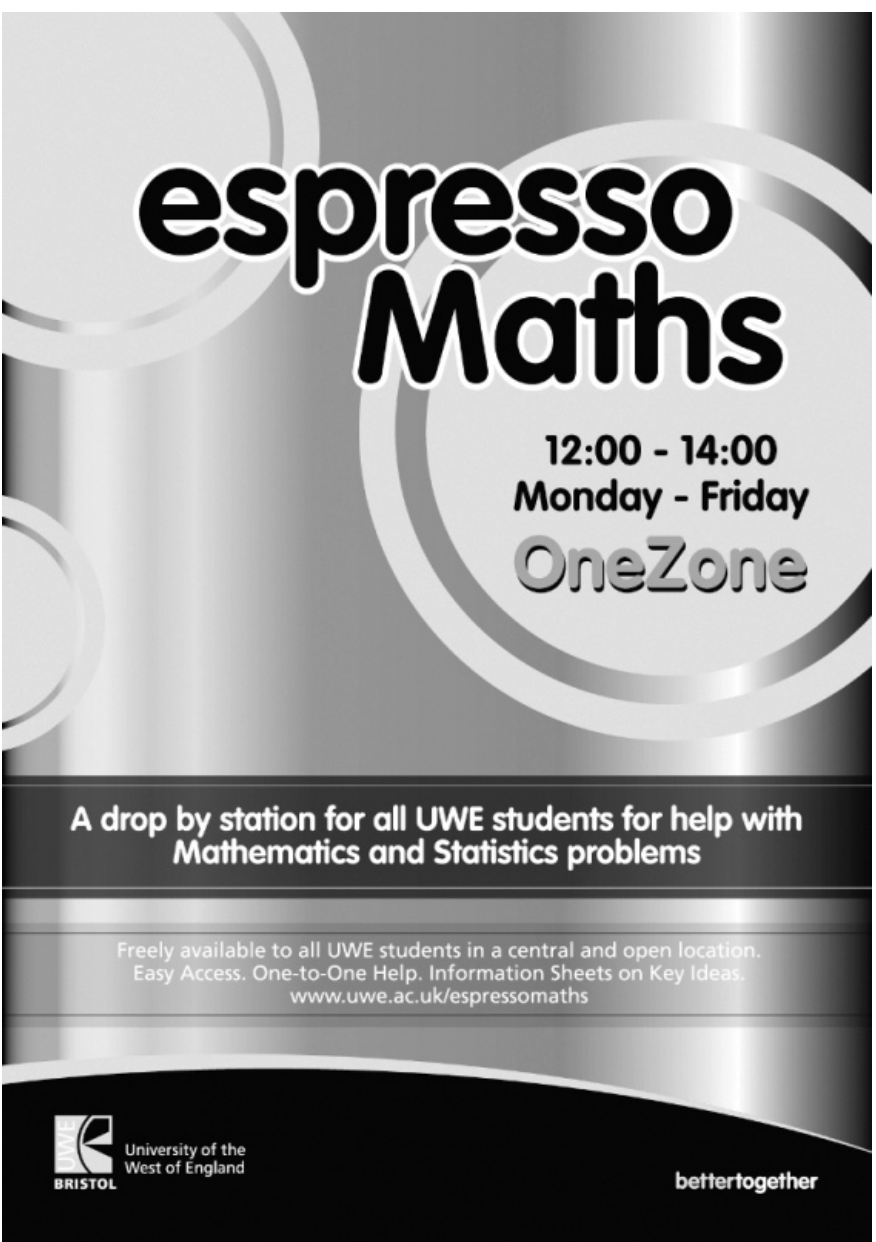

Fig 1 - An example of espressoMaths publicity

\section{Operation}

As mentioned above, the central idea of espressoMaths is that it be a Mathematics and Statistics drop-by station. Thus, it provides extra-curricular support, help and advice, and it does so in a very specific way. For a general historical account of the various different models of Mathematics and Statistics support in Britain and Ireland, see [4].

Drop-by station philosophy has the following ingredients:

1. the physical source of support is located at one of the hearts of the university;

2. the location should be completely open, easy to identify, and easily accessible;

3. the support should be user-friendly and free to all members of the university community;

4. levels of informality and spontaneity should be maximised, e.g., no advanced bookings are available;
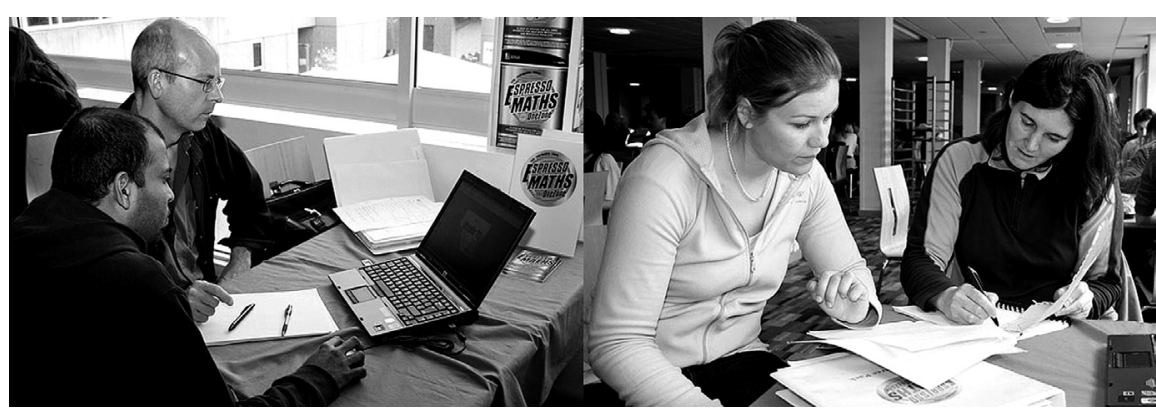

5. any member of the university community may ask for advice on any area of Mathematics or Statistics;

6. the support is provided independently of - and in addition to - all other modes of teaching and learning.

In the case of espressoMaths, how this philosophy translates into action may be described as follows. If a student or staff member needs help with a particular mathematical or statistical problem, then they may pop along to either of the espressoMaths stations. Each of the stations is identified by its red tablecloth covered table (Fig 2). The person presents themselves at the table, and then has a chat with the mathematician or statistician on duty. As well as the academic's professional knowledge and experience being available, the espressoMaths station is equipped with a laptop, thereby providing immediate access to the internet and to several software packages. In addition to being provided with face-to-face/one-to-one help, the person seeking support might be directed to online or to other learning resources for their own self-study. Note that most, but not all, espressoMaths interactions are one-to-one.

Concerning the way in which espressoMaths support fits into the mainstream flow of Mathematics and Statistics teaching and learning, the following remarks may be made. Firstly, depending on the nature of the module, different module leaders encourage different ways of using the drop-by station. For potential student users who require advice concerning material related to a particular module, the general advice is that the usual module resources should be exhausted before the problem is brought along to espressoMaths. Secondly, it should be noted that duty staff are very aware of the various issues associated with students' requesting help with homework, with module coursework, or with dissertations. Indeed, before a student presents their problem in detail, it is established whether or not the issue relates to homework or to some form of assessment. If the query does relate to assessment, then the help provided is not detailed, but rather the student is helped to identify the source of their lack of understanding, and then directed to an appropriate resource for self-study.

It is worthwhile making a couple of final points concerning the way in which espressoMaths operates. Firstly, if the mathematician or statistician on duty is already occupied, a wait of five or ten minutes, or perhaps a return visit may be necessary. Clearly this is not ideal, but it is a consequence of maximising the level of informality of the service. Secondly, the espressoMaths interaction is often two way inasmuch as the duty staff member will receive potentially useful feedback on gaps in Mathematics and Statistics provision on programmes around the University. If such a gap is identified, perhaps after experiencing a sequence of students from the same programme, all of

Fig 2 - An espressoMaths drop-by station in action 
whom have a similar problem, then the information can be fed back to programme directors or to module leaders.

\section{Evaluation}

Evaluation of a given support unit is important, although exactly which aspects can and should be monitored is less clear $[5,6]$.

The primary tool for monitoring the operation of espressoMaths is the daily online log, which is accessed and completed by staff via the website. This log chronicles the way in which the drop-by station is being utilised, and provides us with data for eventual analysis.

We now have over two years' worth of user data, these being broken down according to the nature of the query and also according to discipline. The support seeker's evaluation of the utility of the visit is also part of the data. Although we do not yet have sufficient data for a complete analysis, some preliminary comments can be made. A general remark is that we are experiencing over twice as many visits per annum to the drop-by station as to the Mathematical Resource Centre in its former role as a drop-in support centre.

In the first year of operation, namely 2008-2009, there were 307 recorded visits to espressoMaths, these visits' involving 187 distinct students. In the second year of operation, namely 2009-2010, there were 309 visits, involving 173 distinct students. Fig 3 shows the usage (in terms of total number of visits) during the two complete years 2008-2009 and 2009-2010, broken down according to discipline.

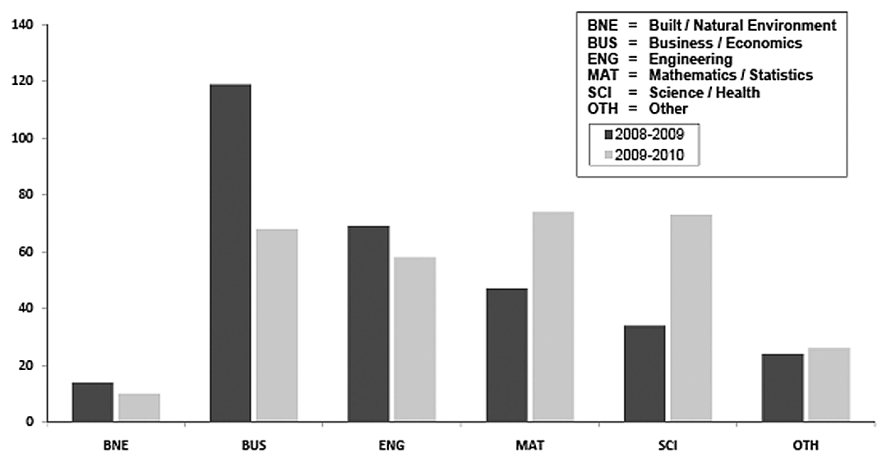

Fig 3 - Number of Visits to espressoMaths, 2008-2010

Another part of our evaluation of the drop-by station took the form of a questionnaire, containing five straightforward questions, and sent by email in early July 2010 to all the 2008-2010 users for whom we had email addresses. Although the response rate to this survey was low, the feedback was overwhelmingly positive. This questionnaire exercise will be repeated at the end of 2010-2011.

\section{Evolution}

Given that espressoMaths has become the main provider of Mathematics and Statistics support within the UWE community, particularly with regard to provision on the Frenchay campus, it is important to discuss how operation of the drop-by station might be taken forward over the next few years. Of course, we will be building on the success that we have achieved to date, and we will be driving forward the drop-by station philosophy.

The main avenues for evolution are as follows.

Firstly, the current provision will be continued and refined in the light of experience. For this, a more complete evaluation of the service will be necessary. For example, one aim is to implement a more efficient queuing system, maintaining as much as possible the informal drop-by aspect of the service.

Secondly, the distributed campus nature of UWE will be better reflected in the way in which the drop-by station is run. As mentioned above, a presence at a second campus - namely Glenside - has recently been established, and this operation will be continued, with a possible increase in weekly frequency, depending on usage during 2010-2011.

Thirdly, in connection with the Glenside station, where training in various health professions is undertaken, we intend to explore ways in which espressoMaths can be integrated more intimately into support for students of nursing and of midwifery.

Fourthly, the ways in which espressoMaths is publicised will be developed. For example, a plan is that there will be a non-staffed satellite presence in the newly developed learning space in the University Library.

Finally, we will continue to interact with and to learn from other providers of Mathematics and Statistics support in order that good practice might be shared and disseminated.

\section{References}

1. Maths Café. Accessed via http://www.port.ac.uk/ departments/academic/maths/mathscafe/ (19 November 2010).

2. espressoMaths. Accessed via http://www.uwe.ac.uk/ espressomaths (19 November 2010).

3. mySkills, University of the West of England'. Accessed via http://www.uwe.ac.uk/library/resources/hub/ (19 November 2010).

4. Samuels, P. and Patel C. (2010) Scholarship in Mathematics Support Services. Journal of Learning Development in Higher Education, Issue 2.

5. Gill, O. and O'Donoghue, J. (2007) Justifying the existence of Mathematics learning support: Measuring the effectiveness of a mathematics learning centre. Proceedings ALM-14: 154-164.

6. Lawson, D., Croft, T. and Halpin, M. (2001) Evaluating and Enhancing the Effectiveness of Mathematics Support Centres. Accessed via http://mathstore.gla.ac.uk/ repository/finalreport.pdf (19 November 2010). 